\title{
Esforço Inovativo e Desempenho Exportador: Evidências para Brasil, Índia e China
}

\author{
Ana Paula Avellar \\ Professora - Universidade Federal de Uberlândia (UFU) \\ Endereço para contato: Av. João Naves de Ávila, 2121 - Campus Santa Mônica \\ Uberlândia - MG - CEP: 38408-100 - E-mail: anaavellar@ie.ufu.br

\section{Luciana Carvalho} \\ Doutoranda - Universidade Federal de Uberlândia (UFU) \\ Endereço para contato: Av. João Naves de Ávila, 2121 - Campus Santa Mônica \\ Uberlândia - MG - CEP: 38408-100 - E-mail: lu_carvalho@hotmail.com
}

Recebido em 23 de outubro de 2012. Aceito em 03 de abril de 2013.

\begin{abstract}
Resumo
O objetivo do artigo é realizar uma investigação empírica acerca da relação entre esforço inovativo e desempenho exportador para uma amostra de empresas industriais do Brasil, Índia e China. Foram utilizados dados por empresa da base Enterprise Survey, organizados pelo Banco Mundial para os anos 2002 e 2003 e estimados modelos probabilísticos. Os resultados encontrados para os três países sugerem que o esforço inovativo medido por novos produtos, gastos em P\&D, índice de tecnologia ou cooperação, aumenta a probabilidade das empresas exportarem. Ademais, para as amostras de empresas de todos os países a participação de capital estrangeiro e os gastos com máquinas e equipamentos aumentam a probabilidade de exportarem. Contudo, nota-se que para as empresas do Brasil, Índia e China, a relação entre esforço inovativo e desempenho exportador é mais tênue do que a observada para empresas de países desenvolvidos.
\end{abstract}

\section{Palavras-Chave}

inovação, exportação, Brasil, Índia e China

\begin{abstract}
The aim of this paper is to develop an empirical investigation about the relation between innovation effort and export performance to a sample of industrial firms from Brazil, India and China. The database used by firms is obtained from World Bank Enterprise Survey 2002 and 2003, and estimated probabilistic models. The findings suggest that a higher innovative effort from Brazil, India and China, measured by new products, R\&D spending, technology index or cooperation, increases the probability of exporting. For all countries, a higher foreign capital and machines and equipments spending increase the probability of exporting. However, this study verify that to Brazilian, Indian and Chinese firms, the linkage between innovative effort and probability to export is weaker than the observed to firms from developed countries.
\end{abstract}

\footnotetext{
- As autoras agradecem o apoio da FAPEMIG para a realização da pesquisa.
} 


\section{Keywords}

Innovation, export, Brazil, India and China

\section{JEL Classificação}

O32, L60

\section{Introdução}

A relação entre inovação e desempenho exportador é um dos principais focos de análise das teorias de comércio internacional. Os estudos pioneiros de Vernon (1961), Posner (1966), Freeman (1968) e Krugman (1979) revelam que a tecnologia pode ser um fator importante da dinâmica dos fluxos de comércio entre países e de seus padrões de especialização. Muitos estudos empíricos acerca da relação entre esforço inovativo e desempenho exportador foram realizados utilizando amostra de empresas de países desenvolvidos (Wakelin, 1998; Sterlacchini, 1999; Kongmanila e Yosk, 2005; Tomiura, 2007; Cassiman e Golovko, 2011). Os resultados encontrados sugerem que o esforço inovativo, medido pelos gastos em $\mathrm{P} \& \mathrm{D}$ e pelo número de inovações, é importante para explicar o desempenho exportador das empresas.

Para países emergentes poucos estudos foram realizados, destacando-se a análise para o caso das empresas no Brasil, China, Filipinas, Índia e Tailândia (Kumar e Siddarthan, 1994; Wignaraja, 2008; Wignaraja, 2011; De Negri, 2005; Arbix, Salerno e De Negri, 2008). Para distintos países, os trabalhos utilizam diferentes medidas de esforço inovativo e apresentam resultados heterogêneos, tornando menos evidente a relação entre esforço inovativo e desempenho exportador das empresas.

O objetivo do trabalho é realizar uma investigação empírica acerca da relação entre esforço inovativo e desempenho exportador para uma amostra de empresas industriais do Brasil, Índia e China. Para tanto, foram utilizados dados por empresa organizados pelo Banco Mundial na base Enterprise Survey, para Brasil (2002), Índia (2002) e China (2003) e como metodologia econométrica estimam-se modelos probabilísticos. Os resultados encontrados para os três países sugerem que o esforço inovativo aumenta a probabilidade das empresas exportarem. Contudo, nota-se que para as empresas do Brasil, 
Índia e China, a relação entre esforço inovativo e desempenho exportador é mais tênue do que o observado para empresas de países desenvolvidos.

Este trabalho pretende contribuir para a literatura empírica acerca da relação entre esforço inovativo e desempenho exportador das empresas em três aspectos: i) realiza um estudo comparado para amostras de empresas de Brasil, Índia e China; ii) utiliza um amplo conjunto de indicadores de esforço inovativo; iii) utiliza uma nova base de dados.

O artigo está organizado em quatro seções, incluindo esta introdução. A segunda seção apresenta o debate teórico e empírico. A terceira seção descreve os dados e os procedimentos metodológicos. $\mathrm{Na}$ quarta seção apresentam-se os resultados econométricos. Por fim, a quinta seção sintetiza as considerações finais.

\section{Inovação Tecnológica e Exportação}

\subsection{Debate Teórico}

A literatura teórica e empírica sobre comércio internacional enfatiza o papel da inovação tecnológica na dinamização dos fluxos de comércio entre países e na configuração de seus padrões de especialização. As primeiras discussões sobre essa temática foram desenvolvidas por Posner (1961) e Vernon (1966).

Posner (1961) desenvolve um modelo com dois países, um líder tecnológico e outro imitador da inovação. Nesse sentido, a inovação tecnológica proporciona poder de monopólio ao país inovador, durante o período de tempo em que o país imitador torna-se capaz de reproduzi-la. Nesse sentido, o trabalho de Vernon (1966) revela que as vantagens competitivas das empresas norte-americanas estariam fortemente determinadas pela sua capacidade inovadora.

Krugman (1989) baseia-se nas análises de Posner e Vernon e desenvolve um modelo Norte-Sul de comércio internacional. O modelo está estruturado em dois países e um fator de produção, sendo o Norte o país inovador, que detém o monopólio temporário dos novos 
produtos, e o Sul o país não inovador, responsável pela produção de tecnologia madura e que necessita de um período de tempo para ser capaz de imitar a nova tecnologia. Nesse sentido, o Norte exporta para o Sul os produtos inovadores e importa produtos de tecnologia madura.

Freeman (1968) apud Xavier et al. (2008) consideram que o referido hiato tecnológico, entre países inovadores e imitadores, pode apresentar longa duração temporal. A partir de um estudo sobre o setor de bens de capital eletrônicos nos Estados Unidos, o autor encontra evidências empíricas que a liderança exportadora desse setor no país está fortemente relacionada ao alto grau de desenvolvimento tecnológico. Entretanto, o autor admite que possa haver limites na análise por se tratar de um estudo para um único setor como o padrão do comércio internacional.

A teoria neo-schumpeteriana do comércio parte de pressupostos distintos das teorias convencionais, pois considera a tecnologia não um bem livremente disponível e que as diferenças tecnológicas explicam a direção e o volume de comércio entre países. Essa abordagem baseia-se em três ideias fundamentais: i) a tecnologia é fenômeno endógeno, portanto, depende do grau de desenvolvimento de cada país; ii) existem relações importantes entre padrão de especialização e crescimento econômico, sendo que a especialização em setores mais inovadores induziria maiores taxas de crescimento; iii) o importante papel das instituições no desenvolvimento tecnológico (Verspagen e Wakelin, 1997 apud De Negri, 2005).

Para Dosi, Pavitt e Soete (1990) a decisão das empresas de realizarem mudança tecnológica é resultado de um processo de busca, a partir do seu estoque de conhecimento e das externalidades. Deste modo, a mudança tecnológica pode ser definida como um processo de aprendizado cumulativo, que desenha as trajetórias das empresas e dos países, com vistas à ampliação da participação no comércio internacional.

De acordo com a literatura teórica, pode-se constatar a existência de uma forte relação entre o ritmo inovativo dos países e sua dinâmica no comércio internacional, de modo que a forte heterogeneidade no grau de inovação entre países inovadores e imitadores acarreta diferenças substanciais na sua forma de inserção comercial. 
Desse modo, interessa a esse estudo investigar empiricamente se o esforço inovativo realizado pelas empresas do Brasil, Índia e China, medido pelos novos produtos inseridos no mercado, pelos gastos em P\&D, pela capacidade de aprendizagem (índice de tecnologia) e pelas atividades de cooperação, está promovendo a competitividade internacional desses países.

\subsection{Evidências Empíricas}

Diversos estudos empíricos, utilizando-se de dados por empresa, encontram evidências de que o esforço inovativo influencia o desempenho exportador de uma empresa. Para Teece (1996), um dos maiores incentivos para as empresas inovadoras se expandirem para mercados estrangeiros refere-se ao aumento de retorno sobre o investimento.

Grande parte desses estudos analisa o caso de empresas de países desenvolvidos e encontra fortes evidências de que o esforço inovador, medido por gastos em $\mathrm{P} \& \mathrm{D}$, número de novos produtos ou redes de cooperação, tem impacto positivo sobre o desempenho exportador das empresas.

Wakelin (1998) investiga a relação entre inovação e exportação de 320 empresas do Reino Unido, dividindo a amostra em inovadoras e não inovadoras. O modelo estimado para explicar o comportamento das empresas quanto à exportação apresenta o resultado de que o número de inovações realizadas no passado tem impacto positivo sobre o comportamento exportador.

Sterlacchini (1999) investiga o comportamento de 4005 empresas em uma região da Itália, por meio da estimação de um modelo probabilístico. $\mathrm{O}$ autor evidencia que a probabilidade da empresa tornar-se uma exportadora é afetada positivamente pelo seu tamanho e pelas atividades inovativas realizadas. Também sobre as empresas italianas, Basile (2001) analisa o comportamento das empresas de todo o país durante os anos 1991 e 1998. Por meio da estimação de um modelo probabilístico, os resultados encontrados sugerem que o esforço inovador coloca-se como um importante fator competitivo e ajuda a explicar o comportamento exportador das empresas na Itália. 
Lachenmaier e Woßmann (2006) analisam o caso das empresas na Alemanha. Os resultados evidenciam a existência de relação positiva entre inovação e esforço exportador, considerando, inclusive, diferentes setores industriais.

Dois importantes estudos apontam evidências para o caso do Japão. Kongmanila e Takahashi (2005) numa análise sobre empresas do setor de vestuário, conclui que as inovações de produto e processo são fatores importantes na determinação do desempenho exportador. Desta forma, o resultado do trabalho mostrou que, para as empresas de vestuário, a inovação, tanto no âmbito do produto quanto no processo de produção, é fonte importante de vantagem competitiva no comércio internacional.

Tomiura (2007) avança no debate e também analisa o comportamento das empresas no Japão quanto à relação entre esforço inovativo da empresa, o estabelecimento de redes de colaboração no exterior (networking) e seu desempenho exportador. Esse estudo amplia a análise Kongmanila e Takahashi (2005) ao considerar 118.300 empresas industriais japonesas, de diferentes setores industriais, agrupadas utilizando-se a classificação de Pavitt (1984). ${ }^{1}$ O resultado encontrado aponta uma relação positiva entre intensidade de P\&D e exportação, especialmente em empresas de pequeno porte.

Desse modo, os resultados encontrados por esse conjunto de estudos apontam evidências consistentes de que em empresas de países desenvolvidos o esforço inovativo influencia positivamente o desempenho exportador das empresas. Destaca-se, entretanto, a existência de poucos estudos na literatura analisando a relação entre esforço inovativo e desempenho exportador para casos de empresas atuantes em países em desenvolvimento. Kumar e Siddarthan (1994) desenvolvem uma análise que pode ser considerada pioneira sobre essa temática para países em desenvolvimento, ao analisar a relação entre inovação e exportação para 640 empresas na Índia durante o período de 1988 a 1990. Os autores encontram evidências de que a inovação tecnológica influencia positivamente o desempenho exportador dos setores industriais.

1 A classificação de Pavitt (1984) divide os setores industriais nos seguintes grupos: produtos primários agrícolas, produtos primários minerais, produtos primários energéticos, indústria agroalimentar, indústria intensiva em outros recursos agrícolas, indústria intensiva em recursos minerais, indústria intensiva em recursos energéticos, indústria intensiva em trabalho, indústria intensiva em escala, fornecedores especializados, indústria intensiva em P\&D. 
Wignaraja (2008) investiga a relação entre inovação e exportação nas empresas do setor de eletrônicos para os casos da China, Tailândia e Filipinas. Os resultados econométricos obtidos utilizando de modelo probabilístico apontam que o esforço inovativo aumenta a probabilidade das empresas do setor de eletrônicos exportarem. Contudo, o autor destaca que a relação entre gastos em $\mathrm{P} \& \mathrm{D}$ e receita de vendas, em nenhum dos casos, mostrou-se estatisticamente significativo.

A análise do autor sugere que para países em desenvolvimento essa variável apresenta pouca representatividade dentre os outros esforços inovativos realizados pelas empresas, tais como a realização de acordos de cooperação com outros parceiros e os esforços de aprendizagem (medido por treinamento, uso de licença de tecnologia do exterior e certificação de qualidade). Esse resultado corrobora as conclusões desenvolvidas pelo estudo realizado por Guan e Ma (2003), em que analisam especialmente o caso da China e encontram evidências semelhantes.

Wignaraja (2011) aprofunda a análise sobre o caso das empresas chinesas, analisando o comportamento do setor automobilístico e de eletrônicos. Os resultados encontrados apontam que o indicador que representa a inovação formal (gasto em $\mathrm{P} \& \mathrm{D} /$ receita) não se mostrou estatisticamente significativo. Contudo, ao se considerar o índice de tecnologia (IT) e os esforços de aprendizagem das empresas, verifica-se que esse tipo de esforço inovativo é o principal determinante da probabilidade de exportação das empresas dos setores analisados.

Alguns estudos no Brasil também mostram a importância da inovação para competitividade das empresas exportadoras. De Negri (2005) conclui em seu estudo que a inovação tecnológica é um fator importante para o desempenho exportador das empresas no Brasil, tanto no que se refere à sua inserção no mercado internacional quanto no aumento dos volumes exportados. Mais que isso, a autora também identifica que empresas inovadoras apresentam um desempenho exportador superior às empresas não inovadoras, especialmente quando a inovação não se restringe à adaptação de produtos e processos. No caso de produtos de menor intensidade tecnológica, as inovações de processo representam um elemento importante para as exportações. Para as exportações de produtos de média intensidade tecnológica, tanto inovações de processo quanto de produto são relevantes. 
Por fim, as exportações brasileiras de produtos de alta intensidade tecnológica parecem não sofrer influência de inovações de produto.

Zucoloto e Toneto Júnior (2005) realizam uma análise empírica sobre o esforço inovativo da indústria brasileira de transformação em comparação com um grupo de países da OCDE (Organização para Cooperação e Desenvolvimento Econômico). Os autores verificam a existência de correlação entre o desempenho tecnológico e o comportamento exportador das empresas brasileiras. Os resultados encontrados apontam que os testes de correlação foram positivos e estatisticamente significativos a $1 \%$, indicando que existe relação positiva entre esforço tecnológico, o desempenho das exportações mundiais e o indicador de vantagens comparativas reveladas na indústria brasileira de transformação.

Arbix, Salerno e De Negri (2008), utilizando dados da Pesquisa de Inovação Tecnológica (Pintec), apontam que esta inovação (compreendida como um produto novo para o mercado) é um ativo importante para a empresa industrial brasileira internacionalizar-se, via investimento direto no exterior. O investimento no exterior, por sua vez, é positiva e fortemente relacionado com a obtenção de preço -prêmio nas exportações. Os resultados comprovam, portanto, que a internacionalização é um passo possível para a empresa inovar e diferenciar produtos.

Com base no debate teórico e no conjunto de evidências apresentados, o estudo contribui para o debate empírico ao testar de maneira pioneira a relação entre esforço inovativo e desempenho exportador, utilizando-se de diferentes indicadores de inovação presentes na literatura, comparando os resultados encontrados para as empresas do Brasil, Índia e China.

\section{Notas Metodológicas}

\subsection{Apresentação dos Dados e Estatística Descritiva}

A análise empírica baseou-se nos dados do Banco Mundial (Enterprises Surveys), para uma amostra de empresas do Brasil (2003), Índia (2002) e China (2003). Enterprises Survey é um le- 
vantamento no nível da firma de uma amostra representativa do setor privado da economia. As pesquisas envolveram um amplo conjunto de temas sobre ambiente de negócios, incluindo as características da empresa, a participação de gênero, acesso ao financiamento, vendas anuais, custos de insumos / trabalho, composição da força de trabalho, licenciamento, infraestrutura, comércio, crime, concorrência, inovação e tecnologia, além de medidas de desempenho. O modo de coleta de dados é por meio de entrevistas. Essas pesquisas têm sido realizadas desde 2002 por diferentes unidades dentro do Banco Mundial. Contudo, desde 2005-06, a maioria dos esforços de coleta de dados tem sido centralizada na Enterprise Analysis Unit. O conjunto de dados de cada país e toda a documentação relevante das pesquisas estão disponíveis ao público no site www.enterprisesurveys.org. As pesquisas são geralmente realizadas em cooperação com as organizações empresariais e agências governamentais que promovem a criação de emprego e crescimento econômico, garantindo a confidencialidade dos entrevistados e das informações.

Inicialmente, faz-se necessário compreender as características das empresas, destacando as possíveis diferenças e semelhanças existentes entre as empresas exportadoras e as não exportadoras dos três países analisados. A Tabela 1 apresenta algumas dessas características. As bases de dados utilizadas são compostas por 1.421 empresas brasileiras (221 exportadoras e 1.200 não exportadoras), 1.378 empresas indianas (295 exportadoras e 1.083 não exportadoras) e 1.489 empresas chinesas (292 exportadoras e 1.197 não exportadoras). De maneira geral, pode-se verificar a existência de um padrão, em que praticamente todos os indicadores das empresas exportadoras são maiores em relação aos das não exportadoras nos três países.

O índice de tecnologia (IT) utilizado nesse estudo baseia-se em Lall (1987, 1992) cujo objetivo é mensurar as capacidades tecnológicas das empresas com base em diferentes tipos de esforços inovativos. É importante ressaltar ainda que esse indicador vem sendo utilizado em diversos estudos empíricos para países emergentes (Lall, 2000; Wignaraja, 2008, 2011), porém, para o caso do Brasil e Índia está sendo usado de maneira pioneira.

Lall (1987, 1992) e Wignaraja (2011) organizam as capacidades tecnológicas das empresas em três grupos: investimento, produção e redes. Para amostra de Brasil, Índia e China essas funções estão iden- 
tificadas nas seguintes variáveis: (i) melhoria de equipamentos, (ii) licenciamento de tecnologia, (iii) certificação de qualidade (ISO), (iv) adaptação e melhoria de produtos, (v) introdução de novos produtos, (vi) atividade de pesquisa e desenvolvimento (P\&D), (vii) subcontratação, (viii) participação em redes de tecnologia. Para cada empresa é atribuída uma pontuação de 1 caso esteja presente essa capacidade tecnológica. A categoria investimento é representada pelas atividades (i) e (ii); a categoria produção é representada por quatro atividades (itens iii a vi); e a categoria redes é representada pelos itens (vii) e (viii). Por fim, o resultado é normalizado para valor entre 0 e l. Este número pode ser interpretado como a pontuação total de capacidades tecnológicas de cada firma.

Quanto ao tamanho das empresas analisadas, as exportadoras destacam-se por serem maiores que as não exportadoras. As empresas exportadoras na China possuem, em média, 584 empregados; no Brasil, possuem cerca de metade do tamanho das chinesas, com uma média de 266 empregados, e na Índia, são ainda menores, com 255 empregados em média. Esse resultado coincide com os resultados encontrados por Tomiura (2007), para empresas japonesas, e para Ganotakis e Love (2010), para empresas do Reino Unido, em que também encontram evidências de que as exportadoras são, em média, dez vezes maiores que as empresas não exportadoras.

No que se refere à participação de capital estrangeiro, verifica-se que $29 \%$ das empresas chinesas exportadoras possuem participação de capital estrangeiro. Dentre os países analisados a participação de capital estrangeiro dentre as exportadoras é, em média, maior na China, seguido do Brasil (18\%) e da Índia (2,4\%). Outro indicador importante para caracterizar as empresas analisadas refere-se à relação entre valor bruto das máquinas e número de funcionários. As empresas exportadoras do Brasil e da China possuem esse indicador maior do que as não exportadoras. A exceção encontra-se no caso indiano, em que para as empresas não exportadoras essa relação é superior às empresas que exportam.

Quanto ao indicador de idade, as exportadoras brasileiras e indianas apresentam, em média, idade de 26,5 e 19,6 anos, respectivamente, em relação às não exportadoras, o que pode indicar que as empresas desses dois países que conseguem se inserir no mercado internacional são mais experientes e maduras. As empresas chinesas, por sua vez, 
diferenciam-se desse padrão, pois as empresas que exportam são mais jovens (em média 12,6 anos) em relação às não exportadoras (em média 17,3 anos).

Tabela 1 - Características das empresas exportadoras e não exportadoras para Brasil, Índia e China

\begin{tabular}{l|c|c|c|c|c|c}
\hline \multirow{2}{*}{ Variáveis } & \multicolumn{2}{|c|}{ Brasil } & \multicolumn{2}{c|}{ Índia } & \multicolumn{2}{c}{ China } \\
\cline { 2 - 7 } & Exporta & Não exporta & Exporta & Não exporta & Exporta & Não exporta \\
\hline Pessoal Ocupado & 266,86 & 77,25 & 255,40 & 45,16 & 584,84 & 332,46 \\
(unidades) & $(498,61)$ & $(154,03)$ & $(783,58)$ & $(141,53)$ & $(1314,96)$ & $(782,91)$ \\
\hline \multirow{2}{*}{$\%$ Capital Estrangeiro } & 16,16 & 2,47 & 2,42 & 0,26 & 28,91 & 6,18 \\
& $(35,62)$ & $(14,68)$ & $(12,25)$ & $(3,26)$ & $(39,53)$ & $(19,17)$ \\
\hline \multirow{2}{*}{ Capital por empregado } & 16,58 & 8,61 & 30,16 & 89,10 & 21,57 & 13,36 \\
& $(71,60)$ & $(7,17)$ & $(381,81)$ & $(280,94)$ & $(1586,01)$ & $(73,66)$ \\
\hline Idade & 26,52 & 16,31 & 19,68 & 15,43 & 12,67 & 17,36 \\
(anos) & $(22,08)$ & $(14,90)$ & $(18,83)$ & $(13,04)$ & $(10,76)$ & $(14,32)$ \\
\hline \% de PO que utiliza & 24,71 & 16,51 & 23,33 & 15,39 & 26,77 & 21,36 \\
Computador & $(21,22)$ & $(16,91)$ & $(25,12)$ & $(22,90)$ & $(28,70)$ & $(26,01)$ \\
\hline Gastos com M\&E & 749,31 & 124,40 & 219,45 & 308,98 & 16497,05 & 1032,69 \\
(US\$) & $(4951,74)$ & $(1704,22)$ & $(1166,86)$ & $(9194,9)$ & $(50375,17)$ & $(63242,96)$ \\
\hline Gastos com P\&D & 180,66 & 24,45 & 230,24 & 2,26 & 321,64 & 311,31 \\
(US\$) & $(690,51)$ & $(161,64)$ & $(4110,73)$ & $(24,21)$ & $(1586,01)$ & $(2717,62)$ \\
\hline \multirow{2}{*}{ Novos Produtos } & 16,52 & 30,44 & 65,55 & 31,79 & 27,78 & 8,13 \\
& $(22,08)$ & $(22,08)$ & $(207,83)$ & $(145,65)$ & $(224,89)$ & $(41,45)$ \\
\hline \multirow{2}{*}{ Índice de Tecnologia } & 0,64 & 0,57 & 0,57 & 0,54 & 0,48 & 0,46 \\
& $(15,12)$ & $(0,1429)$ & $(0,22)$ & $(0,19)$ & $(0,26)$ & $(0,25)$ \\
\hline Total de Empresas & 221 & 1200 & 295 & 1083 & 292 & 1197 \\
\hline
\end{tabular}

Fonte: Banco Mundial (2002, 2003a, 2003b). Elaboração Própria.

As exportadoras também apresentam médias superiores às não exportadoras quanto ao percentual de pessoal ocupado que utiliza computador nos três países analisados. Na China 26,7\% dos trabalhadores das exportadoras usam computador, seguido dos $24,7 \%$ das empresas brasileiras e dos $23,3 \%$ das empresas indianas. No que se refere ao indicador de gastos com máquinas e equipamentos, as empresas exportadoras da China gastam, em média, 16 vezes mais do que as não exportadoras. As empresas brasileiras também gastam com máquinas e equipamentos mais do que as não exportadoras, excetuando-se a esse fato o caso indiano.

Ainda pela Tabela 1 , podem ser observadas três importantes variáveis consideradas em diversos estudos como proxies do esforço inovativo das empresas: gastos em P\&D, desenvolvimento de novos produtos e índice de tecnologia. Quanto aos gastos com P\&D, as empresas exportadoras apresentam montantes, em média, superiores aos das não exportadoras. As empresas brasileiras exportadoras gastaram, 
em média, US\$ 180 milhões em 2003 com atividades de P\&D, montante esse inferior à média das empresas exportadoras chinesas, que gastaram, em média, nesse mesmo ano, US\$ 321 milhões. Ganotakis e Love (2010) no estudo para o Reino Unido também conclui que as empresas exportadoras têm gastos mais elevados com $\mathrm{P} \& \mathrm{D}$, sugerindo que o esforço inovativo (gastos com $\mathrm{P} \& \mathrm{D}$ ) está fortemente relacionado à exportação. Quanto ao desenvolvimento de novos produtos, observa-se a mesma realidade, pois para os três países analisados as empresas exportadoras, em média, desenvolveram, nos três anos anteriores ao ano da pesquisa, mais novos produtos do que as não exportadoras.

No que se refere ao índice de tecnologia, verifica-se que as empresas exportadoras, em relação às não exportadoras, apresentam médias superiores para os três países. Dessa forma, nota-se que as empresas exportadoras apresentam um número maior de capacidades tecnológicas em relação às não exportadoras. No caso do Brasil, as empresas exportadoras apresentaram um IT $12 \%$ superior em relação às não exportadoras. Esse resultado alinha-se com as conclusões obtidas por Wignaraja (2011), que identificou nas empresas chinesas exportadoras, em média, um índice tecnológico 30\% maior do que para as não exportadoras.

A Tabela 2 apresenta outro importante conjunto de variáveis que indicam a frequência de algumas características qualitativas das empresas exportadoras e das não exportadoras para os países analisados. Quanto à realização de programa de treinamento nota-se que as empresas exportadoras possuem um percentual superior às não exportadoras nas empresas dos três países.

Tabela 2 - Características qualitativas das empresas exportadoras e não exportadoras para Brasil, Índia e China. (\%)

\begin{tabular}{l|c|c|c|c|c|c}
\hline \multirow{2}{*}{ Variáveis } & \multicolumn{2}{c|}{ Brasil } & \multicolumn{2}{c|}{ Índia } & \multicolumn{2}{c}{ China } \\
\cline { 2 - 7 } & Exporta & Não exporta & Exporta & Não exporta & Exporta & Não exporta \\
\hline Programa de Treinamento & 85,52 & 62,25 & 34,58 & 17,36 & 96,23 & 92,56 \\
\hline Projetos de Cooperação & 89,59 & 70,75 & 89,15 & 76,08 & 58,22 & 56,89 \\
\hline Alta intensidade tecnológica & 14,93 & 9,58 & 38,98 & 41,27 & 44,52 & 39,77 \\
\hline Média Intensidade tecnológica & 50,23 & 40,50 & 16,27 & 28,53 & 15,07 & 41,60 \\
\hline Baixa intensidade tecnológica & 34,84 & 49,92 & 44,75 & 30,20 & 40,41 & 18,63 \\
\hline Total de Empresas & $\mathbf{2 2 1}$ & $\mathbf{1 2 0 0}$ & $\mathbf{2 9 5}$ & 1083 & $\mathbf{2 9 2}$ & 1197 \\
\hline
\end{tabular}

Fonte: Banco Mundial (2002, 2003a, 2003b). Elaboração Própria. 
No que se refere à realização de projetos de cooperação, as empresas exportadoras participam mais de acordos de cooperação em relação às não exportadoras. Tomiura (2007) também identificou essa relação para as empresas japonesas. Dentro da amostra utilizada, 64\% das empresas exportadoras japonesas participam de um algum tipo de projeto de cooperação. Observa-se, assim, que realizar acordos de cooperação é uma característica predominante em empresas que exportam, e tal resultado sugere que as empresas que se inserem no mercado internacional, via exportação, já se relacionam com parceiros em outros países.

Faz-se necessário também uma compreensão mais aprimorada das características das empresas quanto à intensidade tecnológica. Para fins desse estudo foi utilizada a classificação de intensidade tecnológica proposta por Lall (2000). ${ }^{2}$ Os resultados apontam que a maior parte das empresas exportadoras do Brasil concentra-se em setores de média intensidade tecnológica $(50,23 \%)$. $\mathrm{Na}$ Índia, as empresas exportadoras concentram-se em setores de baixa intensidade tecnológica $(44,75 \%)$, enquanto que na China as empresas exportadoras concentram-se em setores de alta intensidade tecnológica $(44,52 \%)$.

\subsection{Especificação Econométrica}

O modelo estimado nesse estudo é o modelo de regressão probabilística. O modelo probit admite a função de distribuição normal (standard) para expressar a relação não linear entre as probabilidades estimadas da variável dependente e as variáveis explicativas. O modelo probit admite a seguinte hipótese:

$$
I_{i}=b_{0}+b_{1} X_{1 i}+. .+b_{n} X_{n i}
$$

onde $\mathrm{I}_{\mathrm{i}}$ é um índice não observado dependente das variáveis $X_{1 i}, \ldots, X_{n i}$, de tal modo que quanto maior é o valor do índice $I_{i}$, maior é a probabilidade de o indivíduo possuir a característica de interesse.

\footnotetext{
2 A taxonomia desenvolvida por Lall (2000) divide os setores de acordo com atividade tecnológica na manufatura. Para tanto, o autor propõe quatro grupos: setores baseados em recursos, setores de baixa intensidade tecnológica, setores de média intensidade tecnológica, setores de alta intensidade tecnológica. Considerando a base de dados utilizada por este trabalho agruparam-se as empresas nos três últimos setores.
} 
Assume-se também que corresponde um nível limiar (crítico) do índice $I_{i}^{*}$ tal que, se $I_{i}^{*} \leq I_{i}$ o indivíduo possui a característica de interesse, caso contrário não possui esta característica.

Admitindo a hipótese da normalidade, a hipótese de que $I_{i}^{*} \leq I_{i}$ pode ser apresentada do seguinte modo:

$P_{i}=P_{i}\left(Y_{i}=1 \mid X_{1 i}, \ldots, X_{n i}\right)=P\left(I_{i}^{*} \leq I_{i}\right)=P\left(Z_{i} \leq b_{0}+b_{1} X_{1 i}+. .+b_{n} X_{n i}\right)=$

$F\left(b_{0}+b_{1} X_{1 i}+. .+b_{n} X_{n i}\right)$

onde $\mathrm{Z}_{\mathrm{i}} \sim \mathrm{N}(0,1)$.

Desta forma, o modelo utilizado pode ser apresentado pela Equação (3):

$\operatorname{Pr}[X>0]=\propto+\lambda_{1}$ Pessoal Ocupado $+\lambda_{2}$ Idade $+\lambda_{3}$ Capital Estrangeiro + $\lambda_{4}$ Capital $+\beta_{1}$ Treinamento $+\beta_{2}$ Computador $+\beta_{3}$ Gastos Máquinas + $\pi_{1}$ Altatec $+\pi_{2}$ Mediatec $+\mu_{1}$ Novos Produtos $+\mu_{2}$ Gastos P\&D $+\mu_{3}$ $I T+\mu_{4}$ Cooperação

Os coeficientes estimados do modelo probit não têm uma interpretação direta. Para serem comparáveis com os coeficientes estimados do modelo linear têm de ser divididos pelo fator 2,5 (Wooldridge, 2010; Cameron e Trivedi, 2009).

Com intuito de encontrar evidências a respeito da influência da inovação tecnológica sobre as exportações das empresas industriais brasileiras, chinesas e indianas, foram selecionadas algumas variáveis, agrupadas em quatro dimensões: características da empresa, indicadores de capacitação, classificação do setor industrial por intensidade tecnológica e esforço inovador da empresa. O Quadro 1 apresenta as variáveis utilizadas. 
Quadro 1 - Descrição das variáveis

\begin{tabular}{|c|c|}
\hline Nome da Variável & Descrição \\
\hline \multicolumn{2}{|l|}{ A) Características da Empresa } \\
\hline Pessoal Ocupado & Número de pessoal ocupado. \\
\hline Idade & Anos desde a abertura da empresa até 2002 ou 2003. \\
\hline Capital Estrangeiro & $\begin{array}{l}\text { Empresa possui participação de capital estrangeiro. Variável binária: } \\
0 \text { - empresa não possui participação de capital estrangeiro; } \\
1 \text { - empresa possui participação de capital estrangeiro. }\end{array}$ \\
\hline Capital & $\begin{array}{l}\text { Valor Bruto de Máquinas e Equipamentos (US\$) em relação ao número de pessoal } \\
\text { ocupado. }\end{array}$ \\
\hline \multicolumn{2}{|l|}{ B) Indicadores de Capacitação } \\
\hline Treinamento & $\begin{array}{l}\text { Empresa realiza treinamento. Variável binária: } \\
0 \text { - empresa não realiza treinamento; } \\
1 \text { - empresa realiza treinamento. }\end{array}$ \\
\hline Computador & Percentual da força de trabalho que utiliza computador. \\
\hline Gastos Máquinas & Logaritmo dos gastos com máquinas e equipamentos (US\$). \\
\hline \multicolumn{2}{|c|}{ C) Características do Setor Industrial } \\
\hline Alta Tec & $\begin{array}{l}\text { Empresa que pertence a setores de alta intensidade tecnológica de acordo Lall } \\
\text { (2000). Variável binária: } \\
0 \text { - indústria baixa e média baixa intensidade tecnológica; } \\
1 \text { - indústria alta intensidade tecnológica. }\end{array}$ \\
\hline Média Tec & $\begin{array}{l}\text { Empresa que pertence a setores de média intensidade tecnológica de acordo Lall } \\
(2000) \text {. Variável binária: } \\
0 \text { - indústria baixa e alta baixa intensidade tecnológica; } \\
1 \text { - indústria média intensidade tecnológica. }\end{array}$ \\
\hline Baixa Tec & $\begin{array}{l}\text { Empresa que pertence a setores de baixa intensidade tecnológica de acordo Lall } \\
(2000) \text {. Variável binária: } \\
1 \text { - indústria alta e média baixa intensidade tecnológica; } \\
0 \text { - indústria baixa intensidade tecnológica. }\end{array}$ \\
\hline \multicolumn{2}{|c|}{ D) Indicadores de Esforço Inovador } \\
\hline Novos Produtos & Número de novos produtos lançados pela empresa nos últimos 3 anos. \\
\hline Gastos P\&D & Logaritmo dos gastos com pesquisa e desenvolvimento (US\$). \\
\hline Índice de Tecnologia (IT) & $\begin{array}{l}\text { Indicador normalizado das capacidades tecnológicas (Lall, 1987, 1992; Wignaraja, } \\
\text { 2011). Capacidades Tecnológicas selecionadas: } \\
\text { 1. Melhoria de Equipamentos; } \\
\text { 2. Licença para uso de tecnologia; } \\
\text { 3. Certificação ISO; } \\
\text { 4. Melhoria ou Adaptação de Produtos; } \\
\text { 5. Introdução de Novos Produtos; } \\
\text { 6. Atividade de P\&D; } \\
\text { 7. Subcontratação; } \\
\text { 8. Redes de Tecnologia. }\end{array}$ \\
\hline Cooperação & $\begin{array}{l}\text { Empresa participou de programa de cooperação com outros parceiros. Variável } \\
\text { binária: } \\
0 \text { - empresa não realiza cooperação; } \\
1 \text { - empresa realiza cooperação. }\end{array}$ \\
\hline
\end{tabular}

Fonte: Banco Mundial (2002, 2003a, 2003b). Elaboração Própria. 


\section{Resultados da pesquisa}

O objetivo desta seção é verificar se as variáveis explanatórias - características da empresa, indicadores de capacitação, intensidade tecnológica do setor e esforço inovativo - influenciam o desempenho exportador das empresas no Brasil, Índia e China. Como já apresentado anteriormente, para viabilizar essa análise estimou-se modelos de regressão probit, segundo recomendações de Greene (2003). Foi considerada como variável dependente binária a empresa exportar ou não exportar nos três anos anteriores a 2002 para as empresas da Índia e nos três anos anteriores a 2003 para as empresas do Brasil e da China.

Foram estimados quatro modelos com objetivo de analisar os efeitos de diferentes indicadores de esforço inovativo no desempenho exportador das empresas. No primeiro modelo foi utilizado o indicador de resultado do esforço inovativo, medido pelo lançamento de novos produtos, com intuito de verificar seus efeitos sobre a probabilidade da empresa exportar. O segundo modelo utiliza o indicador de esforço inovativo mais formal, segundo a literatura, medido pelos gastos em $P \& D$, com objetivo de analisar se o volume de gastos em P\&D afeta a probabilidade da empresa exportar. No terceiro modelo utiliza-se o índice tecnológico (IT) com intuito de mensurar se o esforço de aprendizagem e as capacitações das empresas afetam a probabilidade de exportar. Por fim, o quarto modelo analisa os efeitos de cooperações empresarias (networking, joint ventures) sobre a probabilidade da empresa exportar. Os resultados obtidos nessas estimações para Brasil, Índia e China estão expressos nas Tabelas 3, 4 e 5 , respectivamente.

Ao analisar os quatro modelos para o caso do Brasil apresentados na Tabela 3 é possível encontrar alguns padrões para as empresas brasileiras. Em todas as estimações verifica-se que dentre as características das empresas, os indicadores de pessoal ocupado, participação de capital estrangeiro e idade apresentaram o sinal esperado, ou seja, seus coeficientes associados são positivos e estatisticamente significativos. Algumas características apontadas na literatura nacional identificam que as empresas estrangeiras têm mais tendência a ser exportadora que as nacionais. De Negri e Acioly (2004) descrevem que a própria natureza das empresas estrangeiras, mais internacionalizadas e com maior inserção no comércio internacional, bem como 
o acesso a canais de comercialização não disponíveis para as empresas domésticas, proporcionam às empresas estrangeiras maiores vantagens competitivas no comércio exterior. Pelo primeiro modelo, pode-se inferir que empresas estrangeiras, atuando num mesmo setor e com os mesmos padrões de inovação das empresas nacionais, possuem uma probabilidade $7,5 \%$ maior de exportar.

Quanto aos indicadores de capacitação, observa-se também a existência de um padrão. As variáveis gastos com máquinas e equipamentos e treinamento possuem coeficientes associados positivos e estatisticamente significativos. Assim, sugere-se, pelos resultados obtidos no segundo modelo, que empresas que realizam esses dois tipos de esforços de capacitação possuem, respectivamente, probabilidade $3,5 \%$ e $11,6 \%$ maior de exportar do que as empresas que não realizam tais esforços. Por fim, as variáveis que determinam as características do setor industrial ao qual a empresa pertence, ou seja, se a participação em setores de alta e média tecnologia afeta a probabilidade da empresa exportar, no caso brasileiro, os resultados dos quatro modelos apontam coeficientes associados positivos e não são estatisticamente significativos.

No que se refere à análise dos quatro indicadores de inovação o resultado é diverso. No primeiro modelo o indicador, medido pelo número de novos produtos lançados nos últimos três anos, apresenta coeficiente associado negativo e não é estatisticamente significativo. Esse resultado indica que novos produtos podem não ser um fator determinante para tornar a empresa brasileira capaz de concorrer em outros mercados, via exportação. Contudo, nos outros três modelos os resultados se assemelham: gastos em $\mathrm{P} \& \mathrm{D}$, índice de tecnologia e cooperação apresentam os sinais esperados dos coeficientes associados positivos e estatisticamente significativos. Esse resultado indica que para o caso das empresas brasileiras o montante gasto em $\mathrm{P} \& \mathrm{D}$, a capacidade tecnológica das empresas e a realização de atividades de cooperação aumentam em 1,6\%, 23,1\% e 11,8\%, respectivamente, a probabilidade das empresas exportarem. 
Tabela 3 - Modelos probit para Brasil

\begin{tabular}{|c|c|c|c|c|c|c|c|c|}
\hline & \multicolumn{2}{|c|}{ Produtos } & \multicolumn{2}{|c|}{ Gasto P\&D } & \multicolumn{2}{|l|}{ IT } & \multicolumn{2}{|c|}{ Cooperação } \\
\hline & Coeficiente & $\begin{array}{l}\text { Efeito } \\
\text { Marginal }\end{array}$ & Coeficiente & $\begin{array}{c}\text { Efeito } \\
\text { Marginal }\end{array}$ & Coeficiente & $\begin{array}{c}\text { Efeito } \\
\text { Marginal }\end{array}$ & Coeficiente & $\begin{array}{l}\text { Efeito } \\
\text { Marginal }\end{array}$ \\
\hline Pessoal Ocupado & $\begin{array}{c}0.00102 \\
(0,00030)^{* *}\end{array}$ & 0.00041 & $\begin{array}{c}0.00076 \\
(0,00037)^{\star *}\end{array}$ & 0.00030 & $\begin{array}{c}0.00083 \\
(0,00036)^{* *}\end{array}$ & 0.00033 & $\begin{array}{c}0.00080 \\
(0,00037)^{\star *}\end{array}$ & 0.00032 \\
\hline $\begin{array}{l}\text { Capital } \\
\text { Estrangeiro }\end{array}$ & $\begin{array}{c}0.00748 \\
(0,00205)^{* * *}\end{array}$ & 0.00299 & $\begin{array}{c}0.00786 \\
(0,00205)^{\star \star *}\end{array}$ & 0.00314 & $\begin{array}{c}0.00773 \\
(0,00202)^{\star * *}\end{array}$ & 0.00295 & $\begin{array}{c}0.00768 \\
(0,00202)^{\star \star *}\end{array}$ & 0.00307 \\
\hline Capital & $\begin{array}{l}-0.00002 \\
(0,00270)\end{array}$ & -0.00001 & $\begin{array}{l}-0.00000 \\
(0,0000)\end{array}$ & -0.00000 & $\begin{array}{r}-0.00016 \\
(0,0019)\end{array}$ & -0.00006 & $\begin{array}{l}-0.00002 \\
(0,00289)\end{array}$ & -0.00001 \\
\hline Idade & $\begin{array}{c}0.00942 \\
(0,00205)^{\star \star *}\end{array}$ & 0.00377 & $\begin{array}{c}0.00964 \\
(0,00275)^{\star \star \star}\end{array}$ & 0.00386 & $\begin{array}{c}0.00999 \\
(0,00276)^{\star \star *}\end{array}$ & 0.00344 & $\begin{array}{c}0.00921 \\
(0,00275)^{\star \star \star}\end{array}$ & 0.00368 \\
\hline Treinamento & $\begin{array}{c}0.30697 \\
(0,10943)^{\star *}\end{array}$ & 0.12279 & $\begin{array}{c}0.29027 \\
(0,11000)^{* *}\end{array}$ & 0.11611 & $\begin{array}{c}0.22970 \\
(0,11943)^{\star * \star}\end{array}$ & 0.09188 & $\begin{array}{c}0.26060 \\
(0,11543)^{\star *}\end{array}$ & 0.10424 \\
\hline Computador & $\begin{array}{c}0.00464 \\
(0,00257)\end{array}$ & 0.00186 & $\begin{array}{c}0.00304 \\
(0.00267)\end{array}$ & 0.00122 & $\begin{array}{c}0.00264 \\
(0,00277)\end{array}$ & 0.00106 & $\begin{array}{l}0.00397 \\
(0,0025)\end{array}$ & 0.00159 \\
\hline Ln Gastos M\&E & $\begin{array}{c}0.09363 \\
(0,18590)^{\star \star \star}\end{array}$ & 0.03745 & $\begin{array}{c}0.08850 \\
(0,18880)^{\star \star \star}\end{array}$ & 0.03540 & $\begin{array}{c}0.08935 \\
(0,01868)^{\star \star \star}\end{array}$ & 0.03303 & $\begin{array}{c}0.09529 \\
(0,18660)^{\star \star *}\end{array}$ & 0.03825 \\
\hline Alta Tecnologia & $\begin{array}{l}0.07248 \\
(0,15176)\end{array}$ & 0.02899 & $\begin{array}{l}0.09191 \\
(0,1498)\end{array}$ & 0.03676 & $\begin{array}{l}0.08674 \\
(0,1498)\end{array}$ & 0.03470 & $\begin{array}{l}0.08670 \\
(0,15046)\end{array}$ & 0.03468 \\
\hline Média Tecnologia & $\begin{array}{l}0.11485 \\
(0,09606)\end{array}$ & 0.04594 & $\begin{array}{l}0.03896 \\
(0,09506)\end{array}$ & 0.01558 & $\begin{array}{l}0.08983 \\
(0,0961)\end{array}$ & 0.35932 & $\begin{array}{c}0.09964 \\
(0,09591)\end{array}$ & 0.03836 \\
\hline Produtos & $\begin{array}{l}-0.00108 \\
(0,0008)\end{array}$ & -0.00043 & & & & & & \\
\hline Gasto P\&D & & & $\begin{array}{c}0.03896 \\
(0,00210)^{\star}\end{array}$ & 0.01598 & & & & \\
\hline $\begin{array}{l}\text { Índice de } \\
\text { Tecnologia }\end{array}$ & & & & & $\begin{array}{c}0,57894 \\
(0,33968)^{*}\end{array}$ & 0.23158 & & \\
\hline Cooperação & & & & & & & $\begin{array}{c}0.29566 \\
(0,11897)^{\star *}\end{array}$ & 0.11826 \\
\hline Pseudo $\mathrm{R}^{2}$ & 0.1846 & & 0.1829 & & 0.1824 & & 0.1848 & \\
\hline Log Likelihood & -500.73 & & -501.80 & & -502.11 & & -500.65 & \\
\hline № Observações & 1421 & & 1421 & & 1421 & & 1421 & \\
\hline
\end{tabular}

Nota: A variável dependente é exportação. As variáveis Gasto P\&D e Gasto Máquinas estão expressas em logaritmo natural. $*, * *, * * *$, significativos a $10 \%, 5 \%$ e $1 \%$, respectivamente. Os erros-padrão são robustos e estão entre parênteses. 
A Tabela 4 apresenta os resultados dos modelos estimados para a amostra de empresas da Índia (1.378 empresas). Ao analisar os quatro modelos do caso da Índia é possível identificar que dentre as características das empresas, que os indicadores de pessoal ocupado e de participação de capital estrangeiro apresentam coeficientes associados positivos e estatisticamente significativos, o que indica que ter grande porte e participação de capital estrangeiro aumenta a probabilidade da empresa exportar, assemelhando-se ao caso das empresas do Brasil.

Quanto aos indicadores de capacitação, observa-se que em todos os modelos as três variáveis - treinamento, computador e gastos com máquinas e equipamentos - possuem coeficientes associados positivos e estatisticamente significativos. Sugere-se com esses resultados que, como mostram os resultados do terceiro modelo, empresas que realizam esses tipos de esforços de capacitação possuem, respectivamente, probabilidade $1,8 \%, 0,2 \%$ e $17,5 \%$ maior de exportar do que as empresas que não realizam tais esforços.

Por fim, as variáveis que determinam as características do setor industrial ao qual a empresa pertence, ou seja, se participar de setores de alta e média tecnologia afeta a probabilidade da empresa exportar, no caso da Índia, os resultados dos quatro modelos apontam coeficientes associados negativos e estatisticamente significativos. Esse resultado sugere que para as empresas desse país pertencer aos setores de alta e média tecnologia diminui a probabilidade da empresa exportar. 
Tabela 4 - Modelos probit para Índia

\begin{tabular}{|c|c|c|c|c|c|c|c|c|}
\hline & \multicolumn{2}{|c|}{ Produtos } & \multicolumn{2}{|c|}{ Gasto P\&D } & \multicolumn{2}{|c|}{ IT } & \multicolumn{2}{|c|}{ Cooperação } \\
\hline & Coeficiente & $\begin{array}{c}\text { Efeito } \\
\text { Marginal }\end{array}$ & Coeficiente & $\begin{array}{c}\text { Efeito } \\
\text { Marginal }\end{array}$ & Coeficiente & $\begin{array}{c}\text { Efeito } \\
\text { Marginal }\end{array}$ & Coeficiente & $\begin{array}{c}\text { Efeito } \\
\text { Marginal }\end{array}$ \\
\hline Pessoal Ocupado & $\begin{array}{c}0,00069 \\
(0,00022)^{* *}\end{array}$ & 0.00028 & $\begin{array}{c}0.00068 \\
(0,00022)^{\star *}\end{array}$ & 0.00027 & $\begin{array}{c}0.00072 \\
(0,0003)^{\star * \star}\end{array}$ & 0.00029 & $\begin{array}{c}0.00070 \\
(0,00023)^{*}\end{array}$ & 0.00028 \\
\hline $\begin{array}{l}\text { Capital } \\
\text { Estrangeiro }\end{array}$ & $\begin{array}{l}0.01650 \\
\left(0,0076^{*}\right.\end{array}$ & 0.00660 & $\begin{array}{c}0.01435 \\
(0,0076)^{\star * *}\end{array}$ & 0.00574 & $\begin{array}{c}0.01722 \\
(0,0077)^{\star *}\end{array}$ & 0.00689 & $\begin{array}{c}0.01581 \\
(0,0074)^{* *}\end{array}$ & 0.00633 \\
\hline Capital & $\begin{array}{l}-0.0000 \\
(0.0000)\end{array}$ & -0.0000 & $\begin{array}{l}-0.0000 \\
(0.0000)\end{array}$ & -0.0000 & $\begin{array}{l}-0.0000 \\
(0.0000)\end{array}$ & -0.0000 & $\begin{array}{l}-0.0000 \\
(0.0000)\end{array}$ & -0.0000 \\
\hline Idade & $\begin{array}{c}0.00320 \\
(0,00286)\end{array}$ & 0.00128 & $\begin{array}{c}0.00164 \\
(0,00286)\end{array}$ & 0.00066 & $\begin{array}{c}0.00275 \\
(0,00284)\end{array}$ & 0.00110 & $\begin{array}{c}0.00342 \\
(0,00284)\end{array}$ & 0.00137 \\
\hline Treinamento & $\begin{array}{c}0.30970 \\
(0,9847)^{\star \star}\end{array}$ & 0.12388 & $\begin{array}{c}0.25810 \\
(0,1005)^{\star * \star}\end{array}$ & 0.10324 & $\begin{array}{c}0.29744 \\
(0,09792)^{\star *}\end{array}$ & 0.11898 & $\begin{array}{c}0.29744 \\
(0,09792)^{*}\end{array}$ & 0.12012 \\
\hline Computador & $\begin{array}{c}0.00530 \\
(0,00175)^{\star *}\end{array}$ & 0.00212 & $\begin{array}{c}0.00450 \\
(0,00174)^{\star *}\end{array}$ & 0.00180 & $\begin{array}{c}0.00536 \\
(0,0016)^{\star * *}\end{array}$ & 0.00214 & $\begin{array}{c}0.00480 \\
(0,001694)^{* *}\end{array}$ & 0.00192 \\
\hline In Gastos M\&E & $\begin{array}{c}0.04338 \\
(0,01361)^{\star * *}\end{array}$ & 0.01735 & $\begin{array}{c}0.31750 \\
(0,01353)^{*}\end{array}$ & 0.12700 & $\begin{array}{c}0.43994 \\
(0,0132)^{\star * *}\end{array}$ & 0.17598 & $\begin{array}{c}0.40190 \\
(0,01323)^{\star}\end{array}$ & 0.16076 \\
\hline Alta Tecnologia & $\begin{array}{c}-0.35030 \\
(0,09381)^{* * *}\end{array}$ & -0.14012 & $\begin{array}{c}-0.38780 \\
(0,09335)^{*}\end{array}$ & -0.15512 & $\begin{array}{c}-0.35225 \\
(0,0920)^{\star \star \star}\end{array}$ & -0.14090 & $\begin{array}{c}-0.35380 \\
(0,09181)^{*}\end{array}$ & -0.14152 \\
\hline Média Tecnologia & $\begin{array}{c}0.05305 \\
(0,01094)^{* * *}\end{array}$ & 0.02122 & $\begin{array}{l}-0.53070 \\
(0,181)^{\star}\end{array}$ & -0.21228 & $\begin{array}{c}-0.53412 \\
(0,1094)^{\star * \star}\end{array}$ & -0.21365 & $\begin{array}{l}-0.52950 \\
(0,1121)^{*}\end{array}$ & -0.21180 \\
\hline Produtos & $\begin{array}{l}0.00033 \\
(0,0002)\end{array}$ & 0.00013 & & & & & & \\
\hline In Gasto P\&D & & & $\begin{array}{c}0.09059 \\
(0,0181)^{\star * *}\end{array}$ & 0.03624 & & & & \\
\hline $\begin{array}{l}\text { Indice de } \\
\text { Tecnologia }\end{array}$ & & & & & $\begin{array}{l}0,27543 \\
(0,2007)\end{array}$ & 0.11017 & & \\
\hline Cooperacao & & & & & & & $\begin{array}{c}0,29164 \\
(0,11216)^{\star *}\end{array}$ & 0.11666 \\
\hline Pseudo $\mathrm{R}^{2}$ & 0.1113 & & 0.1280 & & 0.1111 & & 0.1147 & \\
\hline Log Likelihood & -635.98 & & -623.97 & & -636.07 & & -633.51 & \\
\hline № Observações & 1378 & & 1378 & & 1378 & & 1378 & \\
\hline
\end{tabular}

Nota: A variável dependente é exportação. As variáveis Gasto P\&D e Gasto Máquinas estão expressas em logaritmo natural. ${ }^{*},{ }^{* *},{ }^{* * *}$, significativos a $10 \%, 5 \%$ e $1 \%$, respectivamente. Os erros-padrão são robustos e estão entre parênteses.

No que se refere à análise dos quatro indicadores de inovação o resultado se assemelha parcialmente ao caso brasileiro. No primeiro modelo, o indicador de novos produtos apresenta coeficiente positivo e não é estatisticamente significativo. Esse resultado indica que para 
as empresas indianas, assim como para as brasileiras, novos produtos podem não ser determinantes para tornar a empresa capaz de concorrer com exportação. Dentre os outros três modelos, os gastos em P\&D e cooperação apresentam os sinais esperados dos coeficientes associados positivos e estatisticamente significativos. De acordo com esses resultados, para o caso das empresas indianas o montante gasto em $\mathrm{P} \& \mathrm{D}$ e a realização de atividades de cooperação aumentam em $3,6 \%$ e $11,7 \%$, respectivamente, a probabilidade das empresas exportarem.

A Tabela 5 organiza os resultados dos modelos estimados para o caso da China (1.489 empresas). No que se refere às características das empresas, verifica-se algumas especificidades no caso chinês. Diferentemente do caso brasileiro e indiano, o coeficiente associado à variável pessoal ocupado é positivo e não é estatisticamente significativo, demonstrando que o tamanho da empresa não tem efeitos sobre a probabilidade das empresas da China exportarem. Outra característica da empresa que chama a atenção refere-se à variável idade, pois o coeficiente associado à variável idade é negativo e estatisticamente significativo, indicando que quanto mais anos de atividade a empresa possui menor probabilidade de se inserir no mercado internacional. Outros trabalhos relacionados à China, como Wignaraja (2011) e Guan e Ma (2003), também identificaram a relação inversa entre idade e exportação. Esses autores atribuíram essa relação às mudanças na estrutura produtiva chinesa nas últimas décadas, em que empresas nasceram recentemente com objetivo de produzir para atender ao mercado externo. A semelhança com o caso das empresas do Brasil e Índia está na variável participação de capital estrangeiro, pois em todos os casos o coeficiente associado à variável é positivo e estatisticamente significativo, indicando que as empresas chinesas que possuem capital estrangeiro aumentam em aproximadamente $4,9 \%$ a probabilidade de exportarem.

Quanto aos indicadores de capacitação, observa-se que em todos os modelos das três variáveis - treinamento, computador e gastos com máquinas e equipamentos - apenas o coeficiente associado aos gastos com máquinas e equipamentos é positivo e estatisticamente significativo. Sugere-se com esses resultados que empresas que realizam gastos com máquinas e equipamentos possuem probabilidade em torno de $5,7 \%$ maior de exportar do que as empresas que não realizam tal esforço. 
Assim como no caso da Índia, as variáveis que determinam as características do setor industrial ao qual a empresa pertence, apresentaram, nos quatro modelos, coeficientes associados negativos e estatisticamente significativos. Esse resultado sugere que para as empresas da China pertencer a setores de alta e média tecnologia diminui a probabilidade da empresa exportar quando comparadas a empresas de setores de baixa intensidade tecnológica.

Tabela 5 - Modelos probit para China

\begin{tabular}{|c|c|c|c|c|c|c|c|c|}
\hline & \multicolumn{2}{|c|}{ Produtos } & \multicolumn{2}{|c|}{ Gasto P\&D } & \multicolumn{2}{|c|}{ IT } & \multicolumn{2}{|c|}{ Cooperação } \\
\hline & Coeficiente & $\begin{array}{c}\text { Efeito } \\
\text { Marginal }\end{array}$ & Coeficiente & $\begin{array}{c}\text { Efeito } \\
\text { Marginal }\end{array}$ & Coeficiente & $\begin{array}{l}\text { Efeito } \\
\text { Marginal }\end{array}$ & Coeficiente & $\begin{array}{c}\text { Efeito } \\
\text { Marginal }\end{array}$ \\
\hline Pessoal Ocupado & $\begin{array}{c}0,0000 \\
(0,0004)\end{array}$ & 0.00002 & $\begin{array}{l}0,00005 \\
(0,0004)\end{array}$ & 0.00002 & $\begin{array}{l}0,00005 \\
(0,0004)\end{array}$ & 0.00002 & $\begin{array}{l}0,00005 \\
(0,0004)\end{array}$ & 0.00002 \\
\hline $\begin{array}{l}\text { Capital } \\
\text { Estrangeiro }\end{array}$ & $\begin{array}{c}0,01231 \\
(0,00143)^{* * *}\end{array}$ & 0.00492 & $\begin{array}{c}0,01218 \\
(0,001)^{\star * \star}\end{array}$ & 0.00487 & $\begin{array}{c}0,01218 \\
(0,00143)^{\star \star *}\end{array}$ & 0.00487 & $\begin{array}{c}0,01211 \\
(0,001)^{\star \star \star}\end{array}$ & 0.00484 \\
\hline Capital & $\begin{array}{c}-0,00005 \\
(0,0000653)\end{array}$ & -0.00002 & $\begin{array}{l}-0,00003 \\
(0,00005)\end{array}$ & -0.00001 & $\begin{array}{l}-0,00003 \\
(0,00005)\end{array}$ & -0.00001 & $\begin{array}{l}-0,00003 \\
(0,00005)\end{array}$ & -0.00001 \\
\hline Idade & $\begin{array}{c}-0,01505 \\
(0,0053)^{\star * \star}\end{array}$ & -0.00602 & $\begin{array}{c}-0,01487 \\
(0,0035)^{\star \star \star}\end{array}$ & -0.00595 & $\begin{array}{c}-0,01471 \\
(0,00352)^{\star \star \star}\end{array}$ & -0.00588 & $\begin{array}{c}-0,01479 \\
(0,0035)^{\star \star \star}\end{array}$ & -0.00592 \\
\hline Treinamento & $\begin{array}{l}0.52940 \\
(0,2033)\end{array}$ & 0.21176 & $\begin{array}{c}0,5871 \\
(0,2032)\end{array}$ & 0.23484 & $\begin{array}{c}0,52526 \\
(0,20336)\end{array}$ & 0.21010 & $\begin{array}{c}0,63944 \\
(0,20379)\end{array}$ & 0.25578 \\
\hline Computador & $\begin{array}{r}-0,00165 \\
(0,0016)\end{array}$ & -0.00066 & $\begin{array}{r}-0,00173 \\
(0,0016)\end{array}$ & -0.00069 & $\begin{array}{l}-0,00170 \\
(0,0016)\end{array}$ & -0.00068 & $\begin{array}{r}-0,00168 \\
(0,0016)\end{array}$ & -0.00067 \\
\hline In Gastos M\&E & $\begin{array}{c}0.14100 \\
(0,02396)^{\star \star \star}\end{array}$ & 0.05640 & $\begin{array}{c}0,1438 \\
(0,0257)^{\star * \star}\end{array}$ & 0.05752 & $\begin{array}{c}0,14738 \\
(0,0240)^{\star \star \star}\end{array}$ & 0.05895 & $\begin{array}{c}0,14716 \\
(0,0248)^{\star * *}\end{array}$ & 0.05886 \\
\hline Alta Tecnologia & $\begin{array}{c}-0,59387 \\
(0,10133)^{\star \star \star}\end{array}$ & -0.23755 & $\begin{array}{c}-0,60735 \\
(0,1020)^{\star * *}\end{array}$ & -0.24294 & $\begin{array}{c}-0,61262 \\
(0,1014)^{* * *}\end{array}$ & -0.24505 & $\begin{array}{c}-0,60346 \\
(0,1012)^{\star * \star}\end{array}$ & -0.24138 \\
\hline Média Tecnologia & $\begin{array}{c}-1.20080 \\
(0,1213)^{\star * *}\end{array}$ & -0.48032 & $\begin{array}{l}-1,19550 \\
(0,119)^{\star \star \star}\end{array}$ & -0.47820 & $\begin{array}{c}-1,19870 \\
(0,1202)^{\star \star \star}\end{array}$ & -0.47948 & $\begin{array}{c}-1,19356 \\
(0,1204)^{\star * *}\end{array}$ & -0.47742 \\
\hline Produtos & $\begin{array}{c}0.00085 \\
(0,00030)^{\star *}\end{array}$ & 0.00034 & & & & & & \\
\hline Gasto P\&D & & & $\begin{array}{c}0,00157 \\
(0,01474)\end{array}$ & 0.00063 & & & & \\
\hline $\begin{array}{l}\text { Índice de } \\
\text { Tecnologia }\end{array}$ & & & & & $\begin{array}{c}0,87500 \\
(0,16713)\end{array}$ & 0.35000 & & \\
\hline Cooperação & & & & & & & $\begin{array}{l}-0.03843 \\
(0,08855)\end{array}$ & -0.01537 \\
\hline Pseudo $\mathrm{R}^{2}$ & 0.2073 & & 0.2036 & & 0,2038 & & 0.2037 & \\
\hline Log Likelihood & -584.19 & & -586.94 & & -586.80 & & -586.85 & \\
\hline № Observações & 1489 & & 1489 & & 1489 & & 1489 & \\
\hline
\end{tabular}

Nota: A variável dependente é exportação. As variáveis Gasto P\&D e Gasto Máquinas estão expressas em logaritmo natural. ${ }^{*},{ }^{* *},{ }^{* * *}$, significativos a $10 \%, 5 \%$ e $1 \%$, respectivamente. Os erros-padrão são robustos e estão entre parênteses. 
No que se refere à análise dos quatro indicadores de inovação o resultado se distingue dos resultados do Brasil e Índia. Apenas no primeiro modelo, o indicador de novos produtos apresenta coeficiente associado positivo e estatisticamente significativo. Esse resultado indica que para as empresas chinesas, diferentemente das brasileiras e indianas, novos produtos são determinantes para tornar a empresa capaz de concorrer com exportação. De acordo com esse resultado, o lançamento de novos produtos aumenta em $0,4 \%$ a probabilidade das empresas exportarem.

\section{Considerações Finais}

Quanto às características das empresas, para todos os países analisados, o coeficiente associado à variável participação de capital estrangeiro é positivo e estatisticamente significativo, indicando que a participação de capital estrangeiro afeta positivamente o desempenho exportador das empresas no Brasil, Índia e China.

Quanto aos indicadores de capacitação para as empresas dos três países analisados a variável gastos com máquinas e equipamentos possui coeficiente associado positivo e estatisticamente significativo, indicando que a realização de gastos com máquinas e equipamentos aumenta a probabilidade das empresas exportarem.

Os resultados encontrados para os três países sugerem que o esforço inovador, medido por novos produtos, gastos em P\&D, índice de tecnologia ou cooperação, tem impacto sobre a probabilidade das empresas exportarem. Contudo, não se pode verificar que um único indicador afete a probabilidade das empresas exportarem para os três países ao mesmo tempo. Para as empresas brasileiras gastos com $\mathrm{P} \& \mathrm{D}$, índice de tecnologia e cooperação influenciam positivamente a probabilidade de exportar; para as empresas indianas gastos com P\&D e cooperação influenciam positivamente a probabilidade de exportar; já para as empresas chinesas apenas o indicador de novos produtos influencia positivamente a probabilidade da empresa exportar.

Assim, observa-se que as evidências encontradas não são tão padronizadas quanto nos estudos para empresas de países desenvolvidos, como já apontava Kumar e Siddarthan (1994). Acredita-se que esse 
resultado está de certa maneira relacionado à heterogeneidade da estrutura industrial e do grau de inovatividade de cada país analisado. Conclui-se que para os países emergentes analisados a relação entre esforço inovativo (novos produtos, gastos em P\&D, índice de tecnologia e cooperação) e uma melhor inserção internacional das empresas, via exportações, é mais tênue do que se apresenta nas evidências empíricas para países desenvolvidos.

Por se tratar de um primeiro exercício empírico, o trabalho pretende dar continuidade em investigações futuras, utilizando essa base de dados. Faz-se necessário para seu desenvolvimento um aprimoramento na análise empírica, investigando a existência (ou não) de endogeneidade, o que sugeriria avaliar a necessidade de inclusão de variáveis instrumentais ao modelo.

\section{Referências}

ARBIX, G., SALERNO, M., DE NEGRI, J. A. O impacto da Internacionalização com foco na inovação tecnológica sobre as exportações das firmas brasileiras. DADOS - Revista de Ciências Sociais, Rio de Janeiro, Vol. 48, n. 1, pp. 395-442, 2005.

BANCO MUNDIAL. Enterprise Survey - China. Banco Mundial, 2002.

BANCO MUNDIAL. Enterprise Survey - Brasil. Banco Mundial, 2003a.

BANCO MUNDIAL. Enterprise Survey - Índia. Banco Mundial, 2003 b.

BASILE, R. Export behavior of Italian manufacturing firms over the nineties: the role of innovation. Research Policy, 30, pp. 1185-1201, 2001.

BAUM, C. F. An Introduction to Modern Econometrics Using Stata. Stata Press Publication, 2006.

BLEANEY, M.; WAKELIN, K. Efficiency, innovation and exports. Oxford Bulletin of Economics and Statistics, 64, 3, 2002.

CAMERON, A. C.; TRIVEDI, P. K. Microeconomics Using Stata. Stata Press Publication, 2009.

CASSIMAN, B.; GOLOVKO, E. Innovation and internationalization through exports. Journal of International Business Studies, 42, pp. 56-75, 2011.

COUTINHO, L; HIRATUKA, C.; SABBATINI, R. O desafio da construção de uma inserção externa dinamizadora. Texto produzido para o Seminário Brasil em desenvolvimento, 2003.

DAMIJAN, J. P.; KOSTEVC, C.; POLANEC, S. From Innovation to Exporting or Vice Versa? The World Economy, pp. 374-398, 2010.

DE NEGRI, F. Inovação Tecnológica e Exportações das Firmas Brasileiras. Anais do XXXIII Encontro Nacional de Economia - Anpec. Natal, 2005.

DE NEGRI, J. A.; ACIOLY, L. Novas evidências sobre os determinantes do investimento externo na indústria de transformação brasileira. Brasília. Texto para discussãon.1019. Brasília: IPEA, 2004.

DE NEGRI, J. A.; FREITAS, F. Inovação tecnológica, eficiência de escala e exportações brasileiras. Texto para Discussão. 1044. Brasília: IPEA, 2004. 
DOSI, G.; PAVITT, K, e SOETE, L. G. The Economics of Technical Change and international Trade, London: Harvester Wheatsheaf, 1990.

GANOTAKIS, P.; LOVE, J. H. R\&D, product innovation, and exporting: evidence from UK new technology based firms. Oxford Economic Papers, 2010.

GREENE, W. H. Econometric analysis. New Jersey: Prentice Hall, 5. ed., 2003.

GROSSMAN, G. M.; HELPMAN, E. Technology and Trade. In: GROSSMAN, C.; ROGOFF, K. The Handbook of International Economics, vol. iii, Elsevier Science, 1995.

GUAN, J.; MA, N. Innovative capability and export performance of Chinese firms. Technovation, 23, pp.737-747, 2003.

HIRSCH, S.; BIJAOUI, E.I. R\&D Intensity and Export Performance: A Micro View. Weltwirtschaftliches Archiv 121 (2), pp. 238-251, 1985.

KONGMANILA, X; TAKAHASHI, Y. Inter-Firm Cooperation and Firm Performance: An Empirical Study of the Lao Garment Industry Cluster. International Journal of Business and Management, 2005.

KRUGMAN, P. "Industrial organization and international trade". In:SCHMALENSEE, R.; WILLIG, R. (orgs.), Handbook of Industrial Organization, v.ii, North-Holland: Elsevier Science Publishers, 1989.

KUMAR, N.; SIDDARTHAN, N. S. Technology, Firm Size and Export Behavior in Developing Countries: the case of Indian Enterprises. The Journal of Development Studies, 31, 2, pp. 289-309, 1994.

KUPFER, D.; ROCHA, F. Determinantes Setoriais do Desempenho das Empresas Industrias Brasileiras. In: DE NEGRI, J.; SALERNO, M. (Orgs.) Inovações, Padrões Tecnológicos e Desempenho das Firmas Industriais Brasileiras. Brasília: IPEA, 2005.

LACHENMAIER, S.; WOßMANN, L. Does innovation cause exports? Evidence from exogenous innovation impulses and obstacles using German micro data. Oxford Economic papers, 58, pp. 317-350, 2006.

LALL, S. Learning to industrialize. Basingstoke (UK): MacmillanPress, 1987.

LALL, S. Technological capabilities and industrialization. World Development, 20, 165-186, 1992.

LALL, S. The technological structure and performance of developing country manufactured exports, 1985-1998. QEH Working Paper Series, n. 44, 2000.

PAVITT, K. Sectoral patterns of technical change: towards a taxonomy and a theory. Research Policy, v. 13, 1984.

POSNER, M. International trade and technical change. Oxford Economic Papers 30, 1961.

STERLACCHINI, A. Do innovative activities matter to small firms in non-R\&D intensive industries? An application to export performance. Research Policy, 28, pp. 819-832, 1999.

TEECE, D.J. Firm organization, industrial structure, and technological innovation. Journal of Economic Behavior \& Organization, Vol. 31, 193-224. 1996.

TOMIURA, E. Effects of R\&D and networking on the export decision of Japanese firms. Research Policy 26, pp.758-767, 2007.

VERNON, R. International investment and international trade in the product cycle. Quarterly Journal of Economics, n. 83, 1966.

WAKELIN, K. Innovation and export behavior at the firm level. Research Policy, 26, pp. 829-841, 1998.

WIGNARAJA, G. FDI and Innovation as Drivers of Export Behavior: Firm-level Evidence from East Asia. Working Paper Series, United Nations University - MERIT, 2008-061, pp.01-27, 2008.

WIGNARAJA, G. Innovation, learning, and exporting in China: Does R\&D or a technology index matter? Journal of Asian Economics, 2011.

WOOLDRIDGE, J. M. Econometric Analysis of Cross Section and Panel Data. The MIT Press, 2010. 
XAVIER. C. L.; AVELLAR, A. P.; CUNHA, S. F. Desempenho das Exportações da Indústria Intensiva em P\&D: comparação entre o Brasil e países selecionados no período 1994-2005. Revista Brasileira de Inovação, Rio de Janeiro. pp.409-443, 2008.

ZUCOLOTO, G. F.; TONETO JUNIOR, R. Esforço tecnológico da indústria de transformação brasileira uma comparação com países selecionados. Revista de Economia Contemporânea, Rio de Janeiro, 9(2): 337-365, mai./ago. 2005. 